\title{
Depressão e ansiedade em adolescentes de escolas públicas e privadas
}

\author{
Depression and anxiety in adolescents from public and private schools
}

Joana D’Arc Vila Nova Jatobá', Othon Bastos²

\section{RESUMO}

Objetivo: Identificar prevalência de depressão e de ansiedade em adolescentes matriculados e freqüentando escolas públicas e privadas da cidade do Recife - Pernambuco. Métodos: Por meio de estudo de prevalência, de base populacional, admitindo população de 10.414 alunos das redes pública e privada, prevalência de depressão e transtornos depressivos de 20,3\%, precisão de 5\% e nível de significância de 0,05, a amostragem aleatória e estratificada incluiu 243 alunos, do ensino fundamental e médio de 11 escolas, com idade de 14 a 16 anos. As variáveis foram: idade, religião, tipo, adscrição e porte da escola, escolaridade, tempo de estudo, condição estudantil e laboral, características do núcleo familiar, sexo, estado civil, tipo de pele segundo escala de Fitzpatrick (investigadas por questionário demográfico) e grau de depressão e de ansiedade pelas escalas de Hamilton. Para análise estatística, empregou-se programa Statistical Package for Social Sciences. Resultados: As prevalências de sintomas depressivos expressivos e de ansiedade igualaram-se a 59,9\% e 19,9\%, respectivamente. Foram significativas as associações de sintomas depressivos de intensidade grave com o sexo feminino e a crença religiosa diferentes da corrente do cristianismo. A ideação suicida/tentativa de suicídio foi referida por $34,3 \%$ dos estudantes. Houve associação significativa de ideação suicida com grau leve ou moderado de sintomas depressivos e moderado de ansiedade, assim como de tentativa de suicídio com sintomas depressivos graves, estudo em escola privada e ansiedade severa. Conclusões: A gravidade das características psicopatológicas em uma idade tão jovem está a merecer uma intervenção psicossocial para reduzir suas repercussões para o futuro.

ansiedade, prevalência.

\begin{abstract}
1 Faculdade de Enfermagem Nossa Senhora das Graças, Universidade de Pernambuco (UPE). 2 Departamento de Psiquiatria, Universidade Federal de Pernambuco (UFPE).

Endereço para correspondência: Joana D’Arc Vila Nova Jatobá Rua do Futuro, 575, apto. 201 - Aflitos 52020-220,- Recife/PE Fones: (81) 3241-9124

E-mail: jattoba@hotlink.com.br
\end{abstract}

Objective: To identify depression and anxiety prevalences within adolescents registered and frequenting public and private schools in the city of Recife - Pernambuco. Methods: According to a population based, prevalence study, admitting a population of 10.414 pupils of public and private schools, depression and depressive upheavals prevalence of 20,3\%, precision of $5 \%$ and significance level of 0,05, the random and stratified sampling included 243 pupils, of basic and mean levels of 11 schools, aging from 14 to 16 years old. The variables were: age, religion, school type, adscription and size, scholarship, study duration, scholar and labor condition, characteristic of familiar nucleus, sex, marriage status, skin type according to Fitzpatrick scale (investigated by a demographic questionnaire) and depression and anxiety degrees by Hamilton scales. Statistical analysis was performed with Statistical Package for Social Sciences. Results: The prevalences of expressive depressive symptoms and anxiety were equal to 59.9\% and 19.9\%, respectively. The associations of serious intensity depressive symptoms with female sex and religious belief different from Christianity chain were

Recebido em

$14 / 06 / 2007$

Aprovado em $31 / 08 / 2007$ 


\section{Key-words}

Adolescence, depression, anxiety, prevalence. significant. The suicidal ideation/suicide attempt was related by 34.3\% of students. There was significant association of suicidal ideation with light or moderate depressive symptoms and moderate degree of anxiety, as well as of suicide attempt with: serious depressive symptoms, study in private school and severe anxiety. Conclusions: The gravity of psychopathologic characteristics in a so young age is to deserve a psychosocial intervention to reduce its repercussions for the future.

\section{INTRODUÇÃO}

A adolescência, segundo a Organização Mundial de Saúde (OMS), é o período da vida a partir do qual surgem as características sexuais secundárias e se desenvolvem os processos psicológicos e os padrões de identificação, que evoluem da fase infantil para a adulta. Entre elas, está a transição de um estado de dependência para outro de relativa autonomia. Considera-se adolescência o período de 10 a 19 anos e distingue-se adolescência inicial (entre 10 e 14 anos de idade) e adolescência final (na idade de 15 a 19 anos) (WHO, 2000).

É um período de intensas modificações no desenvolvimento humano, marcado por alterações biológicas da puberdade e relacionado à maturidade biopsicossocial do indivíduo. Desse modo, é identificada como um período de crise, pela experiência de importantes transformações mentais e orgânicas capazes de proporcionar manifestações peculiares em relação ao comportamento normal para a faixa etária. Estas podem, contudo, ser confundidas com doenças mentais ou manifestações comportamentais inadequadas (Peres e Rosenburg, 1998).

Aberastury et al. (1983), ao caracterizarem as modificações psicossociais da adolescência, alertaram que, nessa fase, vários processos de luto são vivenciados. Knobel (2007) denomina o conjunto desses processos de síndrome normal da adolescência (SNA), na qual as principais perdas são: a) do corpo infantil; b) dos pais da infância; c) da identidade e do papel sociofamiliar infantil, que devem ser elaborados para alcançar a posição adulta definitiva. A estas perdas, somam-se os processos de escolha afetiva, a autonomia em relação aos pais, a descoberta progressiva do tornar-se adulto sem a plenitude das aptidões correspondentes e uma verdadeira explosão biológica-humoral peculiar, inerente a esse desenvolvimento (ABEn, 2001).

Se os conflitos próprios dessa fase forem mal conduzidos, podem contribuir para o surgimento de transtornos do humor e, em particular, da depressão (Ranña, 2001).

A concepção tendenciosa à patogenicidade da adolescência, aqui incluída a SNA, é merecedora de crítica, pois estudos epidemiológicos têm revelado que a maioria dos jovens passa pela adolescência sem distúrbios importantes (Offer e Schonert-Reichl, 1992) e que as alterações do humor, o ato de experimentar coisas novas, contestar e testar limites parecem ser características próprias à vida dos adolescentes. Desequilíbrios persistentes não são a regra, e o desenvolvimento normal deve ser distinguido do psicopatológico (Haarasilta, 2003).

A depressão, embora freqüentemente não reconhecida, é comum nessa faixa etária. Habitualmente, é debilitante e crônica no seu curso, com efeitos adversos em longo prazo, especialmente senão for tratada (Birmaher et al., 1996). Assim, a depressão na adolescência vem se constituindo em um crescente e preocupante problema de saúde pública, ainda que poucos estudos epidemiológicos sobre o tema, neste período da vida, tenham sido realizados (Bahls, 2002).

Crianças e adolescentes depressivos costumam apresentar altas taxas de comorbidade com outros transtornos psiquiátricos, em freqüência maior do que em adultos deprimidos (Bahls, 2002). Goodyer e Cooper (1993) destacam que os transtornos depressivos em crianças e adolescentes, em $40 \%$ dos casos, associam-se a comorbidades como transtornos de ansiedade e, em 15\% dos casos, com transtornos de conduta.

Segundo Steinberg e Morris (2001), "nenhuma tentativa de desenvolver uma teoria geral do desenvolvimento adolescente normal encontrou ainda ampla aceitação", mas Haarasilta (2003) considera que as pesquisas sobre o desenvolvimento natural do adolescente são ainda escassas, o que torna plausível admitir a necessidade de mais bem conhecer as modificações psíquicas dessa fase da vida.

O trabalho como enfermeira em diversas especialidades médicas, por longo período, atendendo também a pacientes na fase da adolescência, deixou perceber a importância da saúde mental na qualidade de vida desses pacientes e convidou à reflexão de que os adolescentes não submetidos à internação hospitalar também poderiam ter sua qualidade de vida comprometida por distúrbios da saúde mental, entre eles, a depressão e a ansiedade. Assim motivada, buscou-se na literatura pertinente a base científica dessa hipótese e se identificou a dimensão do problema e a importância do tema, em uma abordagem não hospitalocêntrica, mas social.

É necessário ampliar os estudos sobre depressão em adolescentes em razão da gravidade da doença, aos danos que ela causa à saúde dos indivíduos e a sua incidência crescente. Além disso, julgou-se pertinente associar a investigação da presença da ansiedade à da depressão em 
adolescentes, pelo fato de a ansiedade ser a comorbidade mais freqüente nos transtornos depressivos.

O presente artigo tem o objetivo de identificar as prevalências de depressão e de ansiedade em adolescentes regularmente matriculados e freqüentando escolas públicas e privadas da cidade do Recife.

\section{MÉTODOS}

Por meio de estudo de prevalência, de base populacionalmente, realizado em 11 escolas que ofereciam ensino fundamental II e ensino médio, sendo oito pertencentes à rede pública e três, à rede privada, na cidade do Recife, Pernambuco, foram pesquisados os dados de alunos na faixa etária de 14 a 16 anos.

A amostra foi aleatória e estratificada proporcionalmente, semelhante à empregada no British Association for the Study of Community Dentistry (BASCD) (Pine et al., 1997), adotando-se três estratos: tipo de escola pública ou privada, porte das escolas determinado com base na Lei no 12.944, de 16 de dezembro de 2005, Pernambuco, 2005) e localização da escola segundo a zona (norte ou sul) da cidade.

Empregando-se a sub-rotina Epitable - Sample, do programa EPI-INFO versão 6.04d, o tamanho amostral geral foi calculado com base na prevalência de depressão e de transtornos depressivos em adolescentes igual a 20,3\% (Bahls, 2002), e admitindo-se o efeito desejado igual a 1,00; população igual a 10.414 alunos; precisão desejada de 5\%; e nível de significância igual a 0,05, obteve-se um tamanho estimado de 243 alunos. Foram coletados dados de 242 alunos (Gráfico 1).

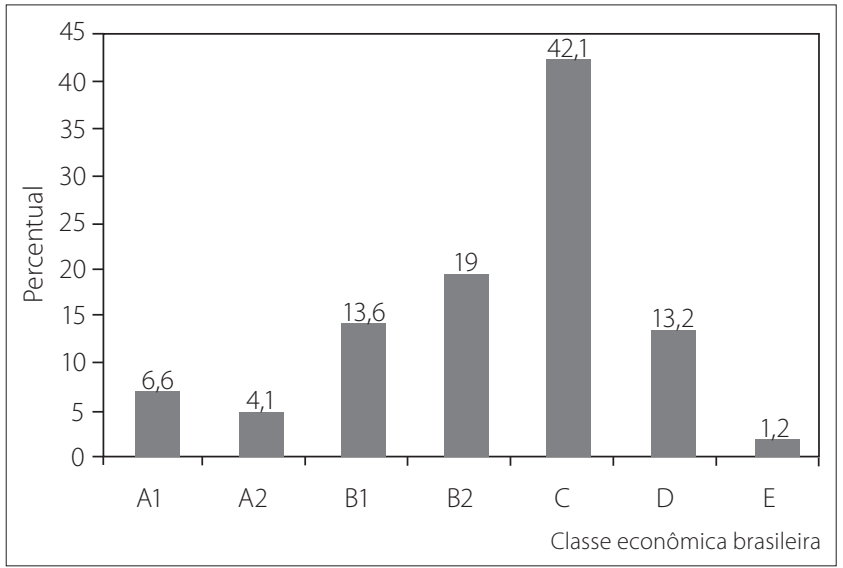

Gráfico 1. Distribuição dos 242 estudantes segundo classe econômica familiar no critério classe Brasil

A aleatorização dos alunos e das escolas foi feita por meio de relação nominal, em ordem alfabética, empregando-se tábua de números aleatórios.

Foram empregados três instrumentos para levantamento de dados: um questionário demográfico, socioeconô- mico e de constelação familiar, a escala de Hamilton para depressão e a escala de Hamilton para ansiedade, ambas validadas no Brasil por Moreno e Moreno (1998) e no Projeto Ambulatório de Ansiedade da Universidade de São Paulo, respectivamente.

No questionário demográfico, socioeconômico e de constelação familiar, foram obtidas as informações referentes a sexo, idade, tipo de pele, estado civil, religião, local de moradia, nível de escolaridade, tempo de estudo, nível de escolaridade do pai e da mãe, estrutura familiar, tipo de família quanto ao responsável, classe econômica e associação da condição estudantil e laboral.

A escala de depressão de Hamilton constituída por 17 itens, com três ou cinco alternativas fechadas convertidas em pontos, adotando-se pontos de corte propostos por Endicott et al. (1981). A escala de ansiedade de Hamilton constituída por 15 itens, com cinco alternativas fechadas convertidas em pontos.

Foram adotadas variáveis de caracterização da amostra e variáveis que contemplam os objetivos da presente pesquisa. Para caracterização amostral, foram adotadas as variáveis: idade, religião, Gerência Regional de Educação (Gere) responsável pela escola, tipo de escola, porte da escola, nível de escolaridade, tempo de estudo, condição estudantil e laboral, local de moradia, tipo de moradia, tipo de abastecimento de água, fornecimento de luz elétrica, tipo de família quanto ao responsável e história de doença mental na família.

Foram classificadas como variáveis de estudo: sexo, estado civil, tipo de pele classificada conforme a escala de fototipos de Fitzpatrick (1988), estrutura familiar, classe econômica ou classe social Brasil com base nos critérios da classificação econômica Brasil da Associação Brasileira de Empresas de Pesquisa (ABEP, 2003), associação da condição estudantil e laboral, grau de sintomas depressivos expressivos, determinado pela escala de Hamilton para depressão (HAM-D), e grau de ansiedade avaliado pela escala de Hamilton para ansiedade (HAM-A),

Os dados foram organizados por meio do programa EPI-INFO versão 6.04d do Center for Disease Control and Prevention (WHO, 2001). A análise dos resultados foi feita utilizando o programa Statistical Package for Social Sciences, versão 13.0. Para a caracterização amostral, foram empregados os parâmetros da estatística descritiva: distribuição de freqüências absolutas e relativas, média, desvio-padrão. Na análise inferencial, foram utilizados os testes estatísticos do qui-quadrado ou exato de Fisher, ambos ao nível de significância de 0,05.

Este estudo foi aprovado pela Comissão de Ética em Pesquisa (CEP) da Universidade de Pernambuco (UPE). A pesquisa não apresentou qualquer forma de conflito de interesses. 


\section{RESULTADOS}

Foram analisados dados de 242 estudantes, na faixa etária de 14 a 16 anos, com média de 14,87 — 1,29 anos; 135 (55,8\%) do sexo feminino e 106 (44,2\%) do masculino. Informaram viver com companheiro(a) 55\% dos estudantes. Questionados quanto à característica da pele no dia seguinte à exposição à radiação solar, dentre os 235 (97,1\%) respondentes, houve predomínio do tipo 4 (139; 59,1\%) sobre o tipo 3 (96; 40,9\%), segundo a classificação de Fitzpatrick.

Os alunos procederam de escolas privadas (29; 12\%) ou públicas (213; 88\%), sob a jurisdição das Gerências Regionais de Educação Norte (88 escolas; 36,4\%) ou Sul (154 escolas; 63,6\%), sendo 42 (17,4\%) de grande porte, 89 (36,8\%) de porte médio e 111 (45,9\%) de porte pequeno, e apresentavam tempo médio de estudo igual a 9,61 $\pm 3,06$ anos. Houve predomínio de alunos cursando o ensino fundamental II (200; 82,6\%), entre a $7^{a}$ e a $8^{a}$ séries, estando 42 (17,4\%) no ensino médio.

Constatou-se que a maioria $(86,6 \%)$ dos pesquisados exercia exclusivamente a ocupação de estudante. Acumulavam outra ocupação além daquela de estudante 32\% (13,4\%) dos adolescentes, quatro (12,5\%) no mercado formal e 28 (87,5\%) no mercado informal, entre os quais predominou o exercício de atividade laboral em ambiente não-familiar.

Quanto à classe econômica das famílias, adotada a classe Brasil, constatou-se predomínio da classe C. Identificouse também que a maioria na amostra pesquisada (56,5\%) percebia mensalmente até 4,5 salários.

O núcleo familiar dos adolescentes estudados caracterizou-se por ser, mais freqüentemente, não tradicional (60,3\%) e patrilinear (51,7\%). Referiram história de doença mental em um membro da família 43\% (17,8\%) dos adolescentes.

Classificados os adolescentes por meio da soma de pontos obtidos na escala de depressão de Hamilton, 95 (40,1\%) obtiveram um total máximo de 7 pontos, tendo sido classificados como ausência de sintomas depressivos, enquanto 147 (59,9\%) estudantes totalizaram 8 a 39 pontos, sendo classificados como presença de sintomas depressivos. Entre os adolescentes com presença desses sintomas, foi constatado grau leve em 99 (40,9\%), seguindo-se grau moderado, acometendo 32 (13,2\%) estudantes, e 14 (5,8\%) casos de sintomas depressivos graves.

Quanto aos aspectos avaliados para classificação da graduação dos sintomas depressivos, identificou-se que $68,6 \%$ dos adolescentes apresentaram humor deprimido, 61,6\% sentimentos de culpa expressos por auto-recriminação ou idéias de culpa e 50,8\% manifestaram agitação, caracterizada por irrequietude e desassossego durante a resposta ao questionário. Menos freqüentemente foram relatados sintomas genitais (perda de libido, sintomas menstruais) (19,8\%), hipocondria $(19,4 \%)$ e perda de peso percebida pelo aluno $(10,7 \%)$ ou referida com base em avaliação que o aluno houvera sido submetido $(8,7 \%)$ (Tabela 1$)$.
Tabela 1. Distribuição dos aspectos avaliados para classificação dos sintomas depressivos dos 242 estudantes (Recife - setembronovembro 2006)

\begin{tabular}{|c|c|c|c|c|}
\hline \multirow{3}{*}{$\begin{array}{l}\text { Aspectos avaliados para } \\
\text { classificação dos sintomas } \\
\text { depressivos }\end{array}$} & \multicolumn{4}{|c|}{ Graduação } \\
\hline & \multicolumn{2}{|c|}{$\begin{array}{c}\text { Ausente } \\
\text { (pontuação }=0 \text { ) }\end{array}$} & \multicolumn{2}{|c|}{$\begin{array}{c}\text { Presente } \\
\text { (pontuação de } 1 \text { a 4) }\end{array}$} \\
\hline & $\mathrm{n}$ & $\%$ & $\mathrm{n}$ & $\%$ \\
\hline Humor deprimido & 76 & 31,4 & 166 & 68,6 \\
\hline Sentimentos de culpa (auto-recriminação) & 93 & 38,4 & 149 & 61,6 \\
\hline Agitação (inquietude e irrequietude) & 119 & 49,2 & 123 & 50,8 \\
\hline Insônia terminal & 128 & 52,9 & 114 & 47,1 \\
\hline Crítica (auto-avaliação de doença) & 135 & 55,8 & 107 & 44,2 \\
\hline Ansiedade somática & 144 & 59,5 & 98 & 40,5 \\
\hline Ansiedade psíquica & 152 & 62,8 & 90 & 37,2 \\
\hline $\begin{array}{l}\text { Trabalhos, estudos e atividades } \\
\text { (interesse, produtividade) }\end{array}$ & 155 & 64,0 & 87 & 36,0 \\
\hline Suicídio (ideação e tentativa) & 159 & 65,7 & 83 & 34,3 \\
\hline Insônia inicial & 165 & 68,2 & 77 & 31,8 \\
\hline Sintomas somáticos - apetite e digestivos & 167 & 69,0 & 75 & 31,0 \\
\hline Sintomas somáticos gerais & 173 & 71,5 & 69 & 28,5 \\
\hline $\begin{array}{l}\text { Retardo (dificuldade de } \\
\text { concentração e lentificação) }\end{array}$ & 176 & 72,7 & 66 & 27,3 \\
\hline Insônia intermediária & 177 & 73,1 & 65 & 26,9 \\
\hline $\begin{array}{l}\text { Sintomas genitais (perda de libido, } \\
\text { sintomas menstruais) }\end{array}$ & 194 & 80,2 & 48 & 19,8 \\
\hline Hipocondria & 195 & 80,6 & 47 & 19,4 \\
\hline $\begin{array}{l}\text { Perda de peso de acordo com a } \\
\text { avaliação do aluno }\end{array}$ & 216 & 89,3 & 26 & 10,7 \\
\hline $\begin{array}{l}\text { Perda de peso aferida em balança em } \\
\text { medidas semanais }\end{array}$ & 221 & 91,3 & 21 & 8,7 \\
\hline
\end{tabular}

É importante ressaltar que 83 (34,3\%) alunos referiram ideação suicida ou mesmo tentativa de suicídio, expressa espontaneamente como: "Eu já tentei suicídio”; "Eu já preparei tudo para me suicidar, mas não tive coragem" ou "Eu já bebi água sanitária" (Tabela 1). A ideação suicida foi mais freqüente em adolescentes do sexo feminino do que no sexo masculino, em uma proporção de 1,88:1,00.

Foi identificada ansiedade de grau leve em 194 (80,2\%) estudantes, seguindo-se o grau moderado, acometendo 27 $(11,2 \%)$ estudantes e o severo em $21(8,7 \%)$ casos.

Quanto aos itens avaliados na escala de ansiedade de Hamilton, constatou-se que foram mais freqüentes: medo, relatado por $24,8 \%$ dos adolescentes, seguindo-se de comportamento ansioso durante a entrevista com a pesquisadora (21,5\%), humor ansioso $(21,1 \%)$ e tensão $(20,7 \%)$. Os sintomas respiratórios, gastrintestinais e geniturinários menos freqüentemente foram referidos $(9,9 \%, 8,7 \%$ e $5,8 \%$, respectivamente) (Tabela 2).

Analisando a associação entre as características dos adolescentes e a graduação de sintomas depressivos, identificou-se que:

1. Os adolescentes com núcleo familiar não-tradicional, comparados àqueles pertencentes a núcleo familiar tradicional, mais freqüentemente referiram sintomas depressivos, mas não houve diferença significativa $(p=0,223)$. 
Tabela 2. Distribuição dos aspectos avaliados para classificação dos sintomas de ansiedade dos 242 estudantes (Recife - setembronovembro 2006)

\begin{tabular}{|c|c|c|c|c|}
\hline \multirow{3}{*}{$\begin{array}{l}\text { Aspectos avaliados para } \\
\text { classificação da ansiedade }\end{array}$} & \multicolumn{4}{|c|}{ Graduação } \\
\hline & \multicolumn{2}{|c|}{ Leve } & \multicolumn{2}{|c|}{ Média a máxima } \\
\hline & $\mathrm{n}$ & $\%$ & $\mathrm{n}$ & $\%$ \\
\hline Medo & 182 & 75,2 & 60 & 24,8 \\
\hline $\begin{array}{l}\text { Comportamento ansioso durante } \\
\text { a entrevista }\end{array}$ & 190 & 78,5 & 52 & 21,5 \\
\hline Humor ansioso & 191 & 78,9 & 51 & 21,1 \\
\hline Tensão & 192 & 79,3 & 50 & 20,7 \\
\hline Dificuldades intelectuais & 196 & 81,0 & 46 & 19,0 \\
\hline Humor deprimido & 198 & 81,8 & 44 & 18,2 \\
\hline Somatizações sensoriais & 200 & 82,6 & 42 & 17,4 \\
\hline Insônia & 202 & 83,5 & 40 & 16,5 \\
\hline Sintomas neurovegetativos & 204 & 84,3 & 38 & 15,7 \\
\hline Somatizações motoras & 207 & 85,5 & 35 & 14,5 \\
\hline Sintomas cardiovasculares & 207 & 85,5 & 35 & 14,4 \\
\hline Manifestações fisiológicas & 211 & 87,2 & 31 & 12,8 \\
\hline Sintomas respiratórios & 218 & 90,1 & 24 & 9,9 \\
\hline Sintomas gastrintestinais & 221 & 91,3 & 21 & 8,7 \\
\hline Sintomas geniturinários & 228 & 94,2 & 14 & 5,8 \\
\hline
\end{tabular}

2. O sexo feminino associou-se à presença de sintomas depressivos $(p=0,006)$, principalmente de intensidade grave $(p=0,003)$.

3. A idade e o tipo da pele não se associaram aos graus de sintomas depressivos ( $p=0,942$ e $p=0,530$, respectivamente).

4. Os adolescentes que declararam professar credo religioso ligado a correntes do cristianismo (referidos como religião católica, evangélica, protestante ou espírita) mais freqüentemente relataram ausência de sintomas depressivos, enquanto aqueles que se declararam adeptos a credo religioso ligado a outras correntes (referidos como religião budista, umbandista e outras) informaram presença de sintomas depressivos, predominantemente de intensidade grave. Essa diferença foi significante $(p=0,005)$.

5. O tipo de escola e a Gere a que ela estava adscrita não se associaram aos sintomas depressivos ( $p=0,798$ e $p=0,685$, respectivamente) (Tabela 3 ).

Dentre os sintomas depressivos, buscou-se detalhar as associações de ideação ou tentativa de suicídio e tipo de escola, graduação dos sintomas depressivos e dos sintomas de ansiedade. A ideação suicida esteve associada a sintomas depressivos leves ou moderados, estudo em escola pública e sintomas moderados de ansiedade, enquanto a tentativa de suicídio associou-se a sintomas depressivos graves, estudo em escola privada e ansiedade severa, sendo todas essas associações significativa ( $p<0,001, p=0,005$ e $p=0,002$, respectivamente) (Tabela 4).
Tabela 3. Distribuição das características dos 242 estudantes segundo graduação dos sintomas depressivos (Recife setembro-novembro 2006)

\begin{tabular}{|c|c|c|c|c|c|c|c|c|c|}
\hline \multirow{3}{*}{$\begin{array}{l}\text { Características } \\
\text { dos } \\
\text { adolescentes }\end{array}$} & \multicolumn{9}{|c|}{ Graduação dos sintomas depressivos } \\
\hline & \multicolumn{2}{|c|}{ Ausentes } & \multicolumn{2}{|c|}{ Leves } & \multicolumn{2}{|c|}{ Moderados } & \multicolumn{2}{|c|}{ Graves } & \multirow{2}{*}{$p$} \\
\hline & $\mathrm{n}$ & $\%$ & $\mathrm{n}$ & $\%$ & $\mathrm{n}$ & $\%$ & $\mathrm{n}$ & $\%$ & \\
\hline Núcleo familiar & & & & & & & & & 0,223 \\
\hline Tradicional & 43 & 38,5 & 8 & 5,6 & 35 & 39,3 & 10 & 12,7 & \\
\hline Não-tradicional & 54 & 58,5 & 6 & 8,4 & 64 & 59,7 & 22 & 19,3 & \\
\hline Sexo & & & & & & & & & 0,029 \\
\hline Feminino & 44 & 45,4 & 11 & 78,6 & 60 & 61,2 & 20 & 62,5 & \\
\hline Masculino & 53 & 54,6 & 3 & 21,4 & 38 & 38,8 & 12 & 37,5 & \\
\hline Idade & & & & & & & & & 0,942 \\
\hline 14 & 38 & 37,3 & 4 & 5,4 & 38 & 38,0 & 13 & 12,3 & \\
\hline 15 & 31 & 29,7 & 6 & 4,3 & 28 & 30,3 & 9 & 9,8 & \\
\hline 16 & 28 & 30,1 & 4 & 4,3 & 33 & 30,7 & 10 & 9,9 & \\
\hline Tipo da pele & & & & & & & & & 0,530 \\
\hline Tipo 2 & 44 & 38,5 & 4 & 5,6 & 33 & 39,3 & 15 & 12,7 & \\
\hline Tipo 4 & 50 & 55,7 & 10 & 8,0 & 63 & 56,9 & 16 & 18,4 & \\
\hline Tipo de credo & & & & & & & & & 0,005 \\
\hline Cristianismo & 88 & 90,7 & 76 & 76,8 & 25 & 78,1 & 8 & 57,1 & \\
\hline Não-cristianismo & 9 & 9,3 & 23 & 23,2 & 7 & 21,9 & 6 & 42,9 & \\
\hline Tipo de escola & & & & & & & & & 0,798 \\
\hline Privada & 10 & 11,6 & 1 & 1,7 & 14 & 11,9 & 4 & 3,8 & \\
\hline Pública & 87 & 85,4 & 13 & 12,3 & 85 & 87,1 & 28 & 28,2 & \\
\hline Gere & & & & & & & & & 0,685 \\
\hline Norte & 35 & 35,3 & 7 & 5,1 & 36 & 36,0 & 10 & 11,6 & \\
\hline Sul & 62 & 61,7 & 7 & 8,9 & 63 & 63,0 & 22 & 20,4 & \\
\hline
\end{tabular}

Tabela 4. Distribuição da graduação de sintomas depressivos, tipo de escola e graduação de sintomas de ansiedade dos 242 estudantes segundo ideação suicida ou tentativa de suicídio (Recife - setembro-novembro 2006)

\begin{tabular}{|c|c|c|c|c|c|c|c|}
\hline \multirow{3}{*}{$\begin{array}{l}\text { Características dos } \\
\text { adolescentes }\end{array}$} & \multicolumn{7}{|c|}{ Graduação dos sintomas de suicídio } \\
\hline & \multicolumn{2}{|c|}{ Ausência } & \multicolumn{2}{|c|}{$\begin{array}{l}\text { Ideação } \\
\text { suicida }\end{array}$} & \multicolumn{2}{|c|}{$\begin{array}{l}\text { Tentativa } \\
\text { de suicídio }\end{array}$} & \multirow[t]{2}{*}{$\mathrm{p}$} \\
\hline & $\mathrm{n}$ & $\%$ & $\mathrm{n}$ & $\%$ & $\mathrm{n}$ & $\%$ & \\
\hline \multicolumn{8}{|l|}{$\begin{array}{l}\text { Graduação de sintomas } \\
\text { depressivos }\end{array}$} \\
\hline Ausente & 89 & 56,0 & 8 & 11,0 & - & - & $<0,001$ \\
\hline Leve & 58 & 36,5 & 35 & 47,9 & 6 & 60,0 & \\
\hline Moderada & 11 & 6,9 & 20 & 27,4 & 1 & 10,0 & \\
\hline Grave & 1 & 0,6 & 10 & 13,7 & 3 & 30,0 & \\
\hline Tipo de escola & & & & & & & 0,005 \\
\hline Privada & 21 & 13,2 & 4 & 5,5 & 4 & 40,0 & \\
\hline Pública & 138 & 86,8 & 69 & 94,5 & 6 & 60,0 & \\
\hline $\begin{array}{l}\text { Graduação de sintomas de } \\
\text { ansiedade }\end{array}$ & & & & & & & 0,002 \\
\hline Leve & 139 & 87,4 & 48 & 65,8 & 7 & 70,0 & \\
\hline Moderada & 13 & 8,2 & 13 & 17,6 & 1 & 10,0 & \\
\hline Severa & 7 & 4,4 & 12 & 16,4 & 2 & 20,0 & \\
\hline
\end{tabular}

Quanto à graduação dos sintomas de ansiedade, foi possível identificar (Tabela 5):

1. Ausência de associação com as características do núcleo familiar ( $p=0,220)$; com o tipo da pele $(p=0,671)$, tipo de credo religioso $(p=0,108)$, tipo de escola $(p=0,192)$ e Gere a que a escola estava adscrita $(p=0,638)$. 
2. Associação significativa entre ser do sexo feminino e apresentar sintomas de ansiedade de graus moderado e severo $(p<0,018)$.

Tabela 5. Distribuição das características dos 242 estudantes segundo graduação dos sintomas de ansiedade (Recife - setembronovembro 2006)

\begin{tabular}{|c|c|c|c|c|c|c|c|}
\hline \multirow{3}{*}{ Variável } & \multicolumn{7}{|c|}{ Graduação da ansiedade } \\
\hline & \multicolumn{2}{|c|}{ Leve } & \multicolumn{2}{|c|}{ Moderada } & \multicolumn{2}{|c|}{ Severa } & \multirow[t]{2}{*}{$p$} \\
\hline & $\mathrm{n}$ & $\%$ & $\mathrm{n}$ & $\%$ & $\mathrm{n}$ & $\%$ & \\
\hline Núcleo familiar & & & & & & & 0,220 \\
\hline Tradicional & 73 & 77,0 & 11 & 10,7 & 12 & 8,3 & \\
\hline Não-tradicional & 121 & 62,4 & 16 & 59,3 & 9 & 42,9 & \\
\hline Sexo & & & & & & & 0,018 \\
\hline Feminino & 103 & 53,1 & 19 & 70,4 & 13 & 61,9 & \\
\hline Masculino & 91 & 46,9 & 7 & 25,9 & 8 & 38,1 & \\
\hline Idade & & & & & & & 0,145 \\
\hline 14 & 75 & 38,7 & 14 & 51,9 & 4 & 19,0 & \\
\hline 15 & 62 & 32,0 & 5 & 18,5 & 7 & 33,3 & \\
\hline 16 & 57 & 29,4 & 8 & 29,6 & 10 & 47,6 & \\
\hline \multicolumn{8}{|l|}{ Cor da pele } \\
\hline Tipo 2 & 80 & 41,2 & 10 & 37,0 & 6 & 28,6 & 0,671 \\
\hline Tipo 4 & 108 & 55,7 & 16 & 59,3 & 15 & 71,4 & \\
\hline Tipo de escola & & & & & & & 0,192 \\
\hline Privada & 20 & 10,3 & 6 & 22,2 & 3 & 14,3 & \\
\hline Pública & 174 & 89,7 & 21 & 77,8 & 18 & 85,7 & \\
\hline Tipo de credo eligioso & & & & & & & 0,108 \\
\hline Cristianismo & 161 & 83,0 & 18 & 66,7 & 18 & 85,7 & \\
\hline Não-cristianismo & 33 & 17,9 & 9 & 33,3 & 3 & 14,3 & \\
\hline Gere & & & & & & & 0,638 \\
\hline Norte & 69 & 35,6 & 12 & 44,4 & 7 & 33,3 & \\
\hline Sul & 125 & 64,4 & 15 & 55,6 & 14 & 66,7 & \\
\hline
\end{tabular}

Identificou-se que a presença de sintomas de ansiedade associou-se significativamente à presença de sintomas depressivos, predominando a ansiedade excessiva e a presença de sintomas depressivos expressivos $(p<0,001)$ (Tabela 6).

Tabela 6. Distribuição dos graus de ansiedade dos 242 estudantes segundo graduação dos sintomas depressivos expressivos (Recife - setembro-novembro 2006)

\begin{tabular}{lcccccc}
\hline \multirow{3}{*}{ Graus de ansiedade } & \multicolumn{5}{c}{ Sintomas depressivos expressivos } \\
\cline { 2 - 3 } \cline { 2 - 3 } \cline { 5 - 7 } & \multicolumn{3}{c}{ Presente } & & \multicolumn{3}{c}{ Ausente } & $\mathrm{p}$ \\
\cline { 2 - 3 } & $\mathrm{n}$ & $\%$ & & $\mathrm{n}$ & $\%$ & \\
\hline Excessiva & 46 & 95,8 & & 2 & 4,2 & $\mathrm{p}<0,001$ \\
Adequada & 99 & 51,0 & & 95 & 49,0 & \\
\hline
\end{tabular}

\section{DISCUSSÃO}

A validade dos resultados de uma pesquisa está condicionada à qualidade dos procedimentos metodológicos que a antecederam (Dersimonian e Kacker, 2006). Por este motivo, antes de analisar os resultados da presente pesquisa cumpre ressaltar alguns aspectos da metodologia adotada com a qual se buscou a obtenção de uma amostra o mais representativa possível dos alunos de escolas públicas e privadas da cidade do Recife, que poderiam ser incluídos. Em primeiro lugar tomou-se por base a estratificação amostral proporcional semelhante à empregada no British Association for the Study of Community Dentistry (BASCD) (Pine et al., 1997), obedecendo ao que preconizam Stroup et al. (2000) no consenso de estudos observacionais epidemiológicos, ao referirem à necessidade de adotar procedimentos amostrais consagrados na realização de pesquisas em populações de características semelhantes.

O BASCD foi realizado nas escolas da Inglaterra, com o objetivo de determinar a prevalência de alterações odontológicas. Tal como a literatura refere ocorrer na depressão e na ansiedade, os problemas odontológicos relacionam-se ao nível socioeconômico e cultural da população pesquisada, já que esses são determinantes dos valores vigentes que interferem diretamente sobre o risco a que os indivíduos aceitam estar submetidos. Por esse motivo, a estratificação da presente pesquisa contemplou escolas das Gere norte e sul, públicas e privadas e, nelas, alunos de todas as séries e níveis, desde que tivessem idade entre 14 e 16 anos, independentemente do sexo.

Apesar de administrativamente terem sido cumpridos todos os procedimentos adequados, para manter a metodologia proposta e solucionar a dificuldade derivada da intolerância de alguns diretores de escola em contribuir para com a pesquisa, as escolas onde não se obteve autorização para coleta de dados foram substituídas por outras na mesma região, com o mesmo porte, pesquisando-se o mesmo número de alunos previamente planejados. A diferença que se observou na presente pesquisa do maior número de escolas na Gere sul do que na norte derivou da distribuição real dessas escolas na cidade do Recife, o que parece sofrer influência do poder aquisitivo, mais do que da distribuição populacional.

Há diversas escalas classificatórias para determinar o grau de severidade dos sintomas de depressão e de ansiedade. Entre essas, está a de Hamilton, que, ao longo do tempo, passou a ser empregada também a pacientes não internados, assim como a pessoas com formas menos severas de depressão e que, portanto, não necessitavam tratamento medicamentoso (Williams, 2001), tendo seu uso sido estendido a adolescentes (Ryder et al., 2005), adotando os mesmos pontos de corte classificatórios de adultos (Endicott et al., 1981).

Apesar da existência de diversas escalas para avaliação de depressão e ansiedade, na época desta pesquisa indubitavelmente a de Hamilton era a mais utilizada mundialmente e, provavelmente, a mais importante, considerando que as demais escalas foram construídas como modificações desta (Calil e Pires, 1998). Por este motivo, a escala de Hamilton tornou-se o padrão-ouro para avaliação, ainda que suscite alguns questionamentos, marcadamente relativos à falta de padronização na aplicação. Williams (2001) afirma que, a 
partir do ano 2000, se tem buscado a criação de um manual de padronização da administração e da pontuação da escala de Hamilton, mas que ela ainda é a mais adequada e a mais utilizada por pesquisadores do mundo todo para fins clínicos e acadêmicos. Apenas em 2007, foi desenvolvida e validada uma escala de depressão para adolescentes, também com base na escala de Hamilton (Revah-Levy et al., 2007).

Do ponto de vista prático, a utilização da escala de Hamilton pode apresentar algumas dificuldades. Pode ser difícil ao avaliado compreender a diferença entre as graduações de intensidade dos sintomas pela escala de Likhert, em virtude dos conceitos pessoais que têm sobre maior ou menor, aumentado ou diminuído, leve, médio, forte e máximo. Esta dificuldade pode estar relacionada à escolaridade do avaliado e a sua classe social, por contribuir para com uma deficiência de vocabulário ou uma capacidade de concentração diminuída, exigindo do avaliador algumas precauções (Möller, 2001).

Quanto ao examinador, podem ocorrer dificuldades oriundas da falta de familiaridade com o teor e a interpretação das respostas ao questionário, assim como em função de sua experiência e de sua capacidade de adequar seu vocabulário às necessidades de compreensão dos avaliandos, mantendo a calma e a discrição necessárias para evitar constrangimentos a ambas as partes. No caso de mais de um avaliador para coleta de dados de um único estudo, pode ocorrer discrepância de conduta na aplicação do questionário, o que também constitui fonte de viés (Möller, 2001).

No presente estudo foram adotadas algumas condutas metodológicas para reduzir a possibilidade de viés. Em primeiro lugar houve um único examinador, a pesquisadora, envolvido na obtenção das respostas ao questionário, que houvera visitado as escolas locais do estudo, o que Ihe possibilitou uma análise prévia da adequabilidade do vocabulário para diálogo com os estudantes durante a coleta de dados, já que tais visitas permitiram avaliar, ainda que grosseiramente, as condições socioeconômicas desses alunos. Esta avaliação prévia foi confirmada pela pesquisa, quando se identificou que os alunos provinham predominantemente da classe Brasil $C$, tendo por parentais indivíduos de baixa escolaridade com núcleo familiar predominantemente não tradicional.

A prevalência de sintomas depressivos expressivos identificados em 59,9\% dos estudantes pesquisados foi maior do que a referida em diversos estudos. Entre eles, o estudo de Bahls, em 2000, na cidade de Curitiba - Paraná, envolvendo 463 alunos de escolas públicas, com idade de 10 a 17 anos, e a prevalência de 13,7\%, determinada por Fonseca et al., em 2005, entre escolares de 7 a 13 anos de idade, no interior do estado de Minas Gerais. A prevalência determinada por Bandim et al. (1995) igualou-se a 12,5\% no Recife. Barbosa e Gaião, em 2001, revelaram 22\% de sintomas depressivos em crianças na Paraíba e Curatolo (2001) obteve taxa de 21\% de crianças com sintomatologia depressiva em São Paulo. No entanto, Espeleta (1988), em 1988, relata taxa variando de $0 \%$ a $64 \%$ entre escolares, em função de diferenças regionais, econômicas, pessoais e de etapas do ciclo da vida. Salle (1999), investigando sintomatologia depressiva em adolescentes de 15 a 17 anos de idade, do ensino médio, em Porto Alegre - Rio Grande do Sul, por meio da escala de Beck, refere 32\% de prevalência. Em pesquisa realizada por Sukiennik et al. (2000) em uma escola do Rio Grande do Sul, a prevalência de sintomatologia depressiva em 150 adolescentes de segundo grau, empregando o Beck Depressment Inventary, igualou-se a $42,7 \%$.

A diferença de prevalência entre o presente estudo e os demais autores citados pode estar relacionada a dois aspectos. Em primeiro lugar cumpre ressaltar que muitos desses adolescentes com sintomas depressivos podem não apresentar transtorno depressivo formal pelos critérios diagnósticos, já que a prevalência deste último varia entre $3 \%$ e $5 \%$. Em segundo lugar deve-se enfatizar que a comparação entre esses estudos pode ser prejudicada em virtude de diferenças regionais, econômicas e culturais existentes entre as populações brasileiras (Baptista e Golfeto, 2000).

A cidade do Recife tem se caracterizado por altas taxas de violência e criminalidade. O mapa da violência 2006, divulgado pela Organização dos Estados Ibero-Americanos para Educação, Ciência e Cultura, aponta que na cidade são mortos, de modo violento, 223,6 jovens de cada 100 mil habitantes, taxa essa muito maior que o da segunda colocada na lista, Vitória - Espírito Santo, em que 64,4 jovens por 100 mil habitantes são mortos por ano.

Lima et al. (2005), em um estudo sobre conglomerados de violência em Pernambuco, referem o aumento das taxas de homicídio na cidade do Recife de 389,8\%, no período de 1998 a 2001, a mais alta do País, e comentam que os focos de violência localizam-se em áreas com condições socioeconômicas precárias, como favelas, nas quais jovens com objetivo de vida convivem com o tráfico e o comércio ilícito de drogas, o roubo, o furto e outros atos de violência que os expõem a riscos e thes exigem a adoção de um novo modo de vida.

Rosa et al., em 2006, ao analisarem a psicanálise diante da realidade, dos ideais e das violências contemporâneas, chamam a atenção para um paradoxo de viver a adolescência, uma fase de ideais e conviver com uma realidade ética e política imposta que os abstém dos dilemas éticos e gera, para além do mal-estar, violências. A isso se associam a necessidade de trabalhar para compor a renda familiar, provir de um núcleo familiar não-tradicional, dispor de pouco apoio social, conviver com o desemprego e por vezes morar sozinho, ter participação limitada em atividades recreativas e consumir álcool e drogas. Parece ter sido esse o 
contexto, no qual os alunos pesquisados referiram sintomas depressivos altamente prevalentes.

Parte dos estudantes que participaram da pesquisa residia na favela do Bode, local assim descrito por Luce Pereira (2006):

[...] jovens criados em lugares insalubres como aquele - que não sabem o que são programas sociais, não vêem a face do Estado, nem das ONG, nem mesmo da Igreja - sem nenhuma referência afetiva, só podem mesmo atribuir a vidas humanas o mesmo valor que atribuem a ratos. Pois são com o que se confundem ali, mergulhados no escuro, na lama, no mau cheiro e nos maus-tratos (Pereira, 2006, p. B12).

Pode-se aventar a hipótese de os adolescentes pesquisados apresentarem sintomas depressivos expressivos em decorrência de estresse pós-traumático pelo que Teixeira e Correia (2002) denominaram fragilidade social, ou seja, por viverem em um ambiente não-saudável, com exposição a abusos e violência, desenvolvendo maior vulnerabilidade aos efeitos do estresse relacionada com auto-estima baixa e ao desenvolvimento de cognições pessimistas que conduzem à diminuição da percepção de controle pessoal sobre as ameaças e sobre os próprios acontecimentos que são vividos.

Dentre os aspectos avaliatórios para classificação dos sintomas depressivos cumpre ressaltar a ideação e a tentativa de suicídio, referidos por 34,3\% dos pesquisados. De acordo com Kasdin e Marciano (1998), a ideação suicida e a tentativa de suicídio são particularmente comuns e podem estar presentes entre 5\% a 20\% dos indivíduos nessa faixa etária, sendo mais comum no sexo feminino, tendo sido atribuídos ao desenvolvimento do pensamento abstrato, por volta dos 12 anos de idade, que traz consigo uma compreensão mais clara do fenômeno da morte, e, conseqüentemente, nos adolescentes depressivos, tanto as idéias de suicídio como as tentativas (Bahls, 2002).

As associações significativa da tentativa de suicídio a sintomas depressivos graves e ansiedade severa, fisiologicamente podem ser explicadas, apesar disso a significância da relação entre estudo em escola privada e ideação ou tentativa de suicídio foi uma constatação para qual Bahls (2003) chamou a atenção ao identificar uma taxa de 30\% de ideação suicida em estudantes adolescentes na cidade de Curitiba, alertando para a associação dela com sintomas depressivos.

Os sintomas depressivos mais prevalentes no presente estudo foram referidos por Bahls (2002) como aspectos clínicos de sintomas depressivos expressivos em adolescentes, podendo estar presentes em até $80 \%$, que se associam sentimentos de auto-recriminação e inquietude.

Quanto à ansiedade, é necessário ressaltar que 80,2\% dos jovens pesquisados apresentaram ansiedade de grau leve com manifestação de um processo motivacional adequado para essa fase de vida, marcada pela descoberta de um mundo novo, à luz de uma compreensão também nova, o que não deve ser preocupante. No entanto, a prevalência de 19,9\% de adolescentes com ansiedade de graus moderado e severo, significativamente associada à presença de sintomas depressivos, pode estar indicando um risco maior de desenvolvimento de transtorno ansioso, cuja prevalência pode variar, em adolescentes, em torno de 4\% (Castillo et al., 2000), podendo, então, ser considerada alta. O entorno de meio ambiente e de convívio pode, também, estar contribuindo com essa prevalência alta por um processo análogo, que parece ter ocorrido na prevalência elevada de sintomas depressivos.

Um achado, que pareceu interessante e indicativo de uma alteração de valores sociais a ser pesquisada com amostras maiores, foi a associação entre sintomas depressivos e credo religioso não-católico, para a qual não se encontrou na literatura referência.

\section{CONCLUSÕES}

Os resultados dessa pesquisa indicam que as características psicopatológicas dos adolescentes estão a requerer que instituições, como a família e a escola, resgatem sua condição de lugar onde o adolescente possa se desenvolver com saúde, abrigado e protegido até que possa melhorar suas condições de vida. A escola e, especialmente, a sala de aula devem ser lugares nos quais as agruras a que estão expostos os adolescentes possam ser minimizadas e o aumento do conhecimento se torne o instrumento que possa efetivamente auxiliá-los a crescer, se desenvolver e evoluir. Para que isso se configure realidade é plausível supor que estes resultados, divulgados entre os professores e os gerentes das escolas pesquisadas possam contribuir para a melhora do adolescente.

\section{REFERÊNCIAS}

ABEn. Associação Brasileira de Enfermagem. Adolescer: compreender, atuar, acolher: Projeto Acolher. Brasília: ABEn,p. 304, 2001.

ABEP. Associação Brasileira de Empresas de Pesquisa. Critério de classificação econômica Brasil. São Paulo: ABEP. 2003.

Aberastury A. et al. Adolescência. Porto Alegre: Artes Médicas. $2^{\text {nd }}$ ed. p. 1-246, 1983.

AMBAN. Ambulatório de Estudo da Ansiedade. Tradução da Escala de Ansiedade de Hamilton. São Paulo, 1985.

Bahls SC. Aspectos clínicos da depressão em crianças e adolescentes. J Pediat, 78(5): 359-366, 2002.

Bahls SC. Sintomas depressivos em estudantes de 10 a 17 anos: um levantamento epidemiológico [tese de dissertação de mestrado em Psicologia da Infância e Adolescência]. Paraná: Universidade Federal do Paraná. Setor de Ciências Humanas, Letras e Artes; 2000.

Bahls SC, Keller APR, Moura CR, Zanona M, Sakiyama RR. Ideação suicida em adolescentes de uma escola pública em Curitiba. Psiq Biol, 11:85-90, 2003.

Bandim JM, Sougey EB, Carvalho TFR. Depression in children: demographic features and symptomatology. J Bras Psiquiatr, 44(1): 27-32, 1995. 
Baptista CA, Golfeto JH. Prevalência de depressão em escolares de 7 a 14 anos. Rev Psiq Clin, 27:5, 2000.

Barbosa GA, Gaião AA. Apontamentos em psicopatologia infantil. João Pessoa: Idéia. 2001.

Birmaher B, Ryan N, Williamson D, Brent DA, Kaufman J, Dahl RE, Perel J, Nelson B. Childhood and adolescent depression: a review of the past 10 years. Part I. J Am Acad Child Adolescent Psychiatr, 35(11): 1427-1439, 1996.

Calil HM, Pires MLN. Aspectos gerais das escalas de avaliação de depressão. Rev Psiq Clin, 26(5):240-24, 1998.

Castillo ARGL, Recondo R, Asbahr FR, Manfro GG. Transtornos de ansiedade. Rev Bras Psiquiatr, 22(suppl II): 20-23, 2000.

Curatolo E. Estudo da sintomatologia depressiva em escolares de sete a doze anos de idade. Arquivos de Neuropsiquiatria, XVI Congresso Brasileiro de Neurologia e Psiquiatria Infantil. Campinas, p. 215, 2001.

Dersimonian R, Kacker R. Randon-effects model for meta-analysis of clinical trials: an up date. Contemporary Clinical Trials, 1-10, 2006.

Endicott J, Cohen J, Nee J, Fleiss J, Sarantakos S. Hamilton depression rating scale. Arch Gen Psych 38:98-103,1981.

Espeleta L. Epidemiologia de la depression infantil en el Municipio de Barcelona. Rev Psiq Faculdade de Medicina de Barcelona, 15:255-268, 1988.

Fitzpatrick TB. The validity and practicality of sun-reactive skin types I through VI. Arch Dermatol, 124: 869-871, 1988

Fonseca MHG, Ferreira RA, Fonseca SG. The prevalence of depression symptoms in scholars. Pediatr São Paulo, 27(4):223-232, 2005.

Goodyer I, Cooper PJA. community study of depression in adolescent girls II. The clinical features of identified disorders. Br J Psychiatry, 163: 374-380, 1993.

Haarasilta L. Major depressive episode in adolescence and young adults - a nationwide epidemiological survey among 15-24 years old. 2003. 95f. Academic Dissertation (Department of Psychiatry, Medical Faculty). Helsinki: University of Helsinki.

Kasdin A, Marciano P. Childhood and adolescent depression. In: Mash, R. B. Treatment of childhood disorders. $2^{\text {nd }}$ ed. New York: Guilford Press, 211-248, 1998.

Knobel M. Adolescência normal - componente psicológico. Disponível em: http://www.mauricioknobel.net/adonormal.htm. Acesso em: 16/01/2007.

Lima MLC, Ximenes RAA, Feitosa CL, Souza ER, Albuquerque MFPM, Barros MDA. et al. Conglomerados e violência em Pernambuco, Brasil. Rev Panam Salud Publica, 18(2):122-128, 2005

Möller HJ. Methodological aspects in the assessment of severity of depression by the Hamilton Depression Scale. Eur Arch Psychiatry Clin Neurosci, 251(suppl 2):II/13-II/20, 2001.

Moreno RA, Moreno DH. Escalas de depressão de Montgomery \& Asberg (MADRS) e de Hamilton (HAM-D). Rev Psiq Clín. 1998.
Offer D, Schonert-Reichl KA. Debunking the myths of adolescence: findings from recent research. J Am Acad Child Adolesc Psychiatry, 31:1003-1014, 1992.

Pereira L. Ratos e homens. Jornal Diário de Pernambuco. Vida Urbana. B12. 25 de novembro de 2006.

Peres F, Rosenburg CP. Desvelando a concepção de adolescência/adolescente presente no discurso da saúde pública. Saude e Sociedade, 7(1): 53-86, 1998.

Pernambuco. Governo do Estado. Lei n 12.944, de 16 de dezembro de 2005. Introduz modificações na Lei nº 12.242 de 28 de junho de 2002, e dá outras providências. Recife: Diário Oficial de 17/12/05.

Pine CM, Pitts NB, Nugent ZJ. British Association for the study of community dentistry (BASCD) quidance on sampling for surveys of child dental health A BASCD coordinated dental epidemiology programme quality standard. Community Dental Health, 14(suppl 1): 10-17, 1997.

Ranña W. Infância e adolescência - enfoque psicodinâmico. In: Fráquas RJ, Figueiró JAB. Depressões em medicina interna e em outras condições médicas - depressões secundárias. São Paulo: Atheneu 401-405, 2001.

Revah-Levy A, Birmaher B, Gasquet I, Falissard B. The adolescent depression rating scale (ADRS): a validation study. BMC Psych, 7:2-10, 2007

Rosa MD, Carignato TT, Berta SL. Ética e política: a psicanálise diante da realidade, dos ideais e das violências contemporâneos. Agora (Rio de Janeiro), IX(1):35-48, 2006.

Ryder AG, Goldbloom DS, Schüller DR, Bagby RM. Use of psychometric principles in evaluating the Hamilton Depression Rating Scale. Direct Psych, 25:241:256, 2005.

Salle E. Estudo da sintomatologia depressiva em adolescentes de 15 a 17 anos de Il grau de Porto Alegre, através das escalas BDI, CRS e CES-D. 1999. Tese (Dissertação de Mestrado) - Universidade Federal do Rio Grande do Sul, Porto Alegre, RS.

Steinberg L, Morris AS. Adolescent development. Annu Rev Psychol, 52: 83-110, 2001.

Stroup DF, Berlin JA, Morton SC, Olkin I, Williamson GD, Rennie D. Moher D, Becker BJ, Sipe TA, Thacker SB. Meta-analysis of observational studies in epidemiology. JAMA, 283(15):2008, 2000.

Sukiennik PB. Segal J, Salle E, Piltcher RB, Teruchkin B, Preussler CM. Implicaciones de la depresión del riesgo de suicidio en la escuela durante la adolescencia. Adolesc Latinoam, 2:1, 2000.

Teixeira JAC, Correia AR. Fragilidade social e psicologia da saúde: um exemplo de influências no contexto sobre a saúde. Anal Psicol, 3(XX):359-365, 2002.

WHO. World Health Organization. EPI INFO versão 6.04d. Janeiro 2001. Centers for Disease Control and Prevention.

WHO. World Health Organization. What about boys? A literature review on the health and development of adolescent boys. In: WHO. Sexuality, reproductive health and fatherhood. cap. 3, Genévé: WH0, p. 29-40, 2000.

Williams JBW. Standardizing the hamilton depression rating scale: past, present, and future. Eur Arch Psychiatry Clin Neurosci, 251(suppl 2):II/6-1I/12, 2001. 